\title{
The Treatment of Leprosy.
}

Dr. R. G. Cochrane.

A Paper read at the Calcutta Leprosy Conference on 27th March, 1933.

(A) Standard treatment for centres with no facilities for experiment.

In considering the best type of treatment to lay down in centres where it is impossible to experiment, the following four principles should as far as possible be adhered to :-

(1) Relative efficacy.

(2) Cheapness.

(3) Relative painlessness.

(4) Ease of administration.

(1) Relative efficacy.

As will be said later, it is extremely difficult to evaluate the results of any of the special lines of treatment in leprosy, but there are certain preparations which have been used long enough for us to be able to say that they are, as far as we know at present, the most effective drugs in the treatment of leprosy. In order of their efficacy I think most workers would place them thus:-

(i) Iodised or creosoted esters.

(ii) Pure hydnocarpus oil and creosote.

(iii) Alepol or the sodium salts of the hydnocarpic acids.

(2) Cheapness.

One must always remember when recommending a certain line of treatment to a centre of the type we are considering, that the cost of the remedy is an important factor. Therefore, as the iodised esters are the most 
expensive, it might be better to use hydnocarpus oil, unless the centre is sufficiently well equipped to make its own esters, or to produce them at inexpensive rates from a neighbouring source. As we are considering Indian conditions where the oil is easily obtainable, one hardly needs to consider alepol. The advantage of the latter preparation is that the drug occupies a small bulk and it can easily be transported, whereas the pure oil is more difficult to transport. Again, alepol is easily injected, being a watery solution and easily made up ; all that is required is distilled water and a little carbolic acid.

(3) Relative painlessness.

This factor is of most importance, for if a drug is painful, patients will be discouraged to attend the dispensary. The least painful of the drugs is the hydnocarpus oil ; esters are very slightly more painful, and alepol is liable to be the most painful.

(4) Ease of administration.

The methods of administration are well known and are as follows :-

(a) Intravenous.

(b) Intramuscular.

(c) Subcutaneous.

(d) Intradermal.

The intravenous route is little used, and the two chief methods are intradermal and subcutaneous. The method of subcutaneous infiltration being easy and comparatively painless is often the method of choice. The intradermal method is the most painful and it takes a longer time to administer compared to the subcutaneous route. Further there is the question whether intradermal methods alone are as efficacious as intradermal injections combined with subcutaneous and intramuscular injections. I should personally recommend the subcutaneous route as a routine, with the painting of the patches with trichlor-acetic acid. In cases with infiltrated erythematous patches and in those patches where bacilli can be demonstrated, I should suggest that these should be given intradermal injections.

\section{(B). Experimental Treatment with suitable staff and equipment.}

This is a subject which is difficult to deal with in a limited space, and therefore I can only express thoughts running through my mind. 
Leprosy treatment has tended to follow along one line. By this I mean that one line or method of treatment tends to be pushed to the exclusion of others. The method now recommended is intradermal injection, while previously it was subcutaneous infiltration plus trichlor-acetic acid. Effective as intradermal injections are, is it a sound policy to confine oneself largely to this method alone? It has been suggested that all we do in intradermal injections is to scar up the local lesion leaving the deep foci untouched. It is known that the ester or oil remains in the tissue for months after injections. Have properly controlled experiments ever been done to show that intradermal injections alone are as efficacious as subcutaneous, combined with intradermal? Further, what is the optimum of dose of ester or oil? There is a tendency not to exceed 5 or 6 c.c. of the remedy because of the pain of the injection, but it has been known for some time that those patients who can take large quantities of oil by mouth usually improve. Therefore I suggest that perhaps our doses tend to be too small. It would therefore be helpful to have evidence as to the optimum dose of the drugs used. Further, have there been any properly controlled experiments giving evidence that intradermal injections are superior to trichlor-acetic acid? If there have not, then the pain of the intradermal method is a decided drawback.

The tendency to adhere to the chaulmoogra oil derivatives has been marked. There have, however, been one or two breakaways from well-trodden paths, and institutions with suitable equipment should be encouraged to experiment along profitable lines. Two lines of treatment suggest themselves to me. The first is the aniline dyes. Dr. Ryles reports favourably on the use of brilliant green, and is now using Bonny's blue, which is a mixture of brilliant green and crystal violet. It is well known that it is possible by the eosin and other analine dyes to sensitive living cells and to cause by the action of visible light abnormal conditions, similar to those produced by ultra-violet radiation.

A vast field of experimentation is opened out here; further, there is a considerable amount of ultra-violet radiation which can be used in the sun's rays. This suggestion is thrown out in order to open up the way for experimentation, by the intradermal injections of aniline dyes and the deliberate exposure of the areas to the sun's rays.

Although the therapeutics of leprosy are much more satisfactory than ten years ago, workers in well-equipped 
institutions should ever be on the look out for other remedies, and if the indigenous systems of medicine recommend any particular remedy, this should afford a basis for trial. Dr. Rao mentioned the use of esters made from Pongamia glabra.

These are some lines along which experiments might be initiated, and institutions sufficiently well equipped should be ready to try out lines of experiments which appear to be fruitful. On the other hand, some supervision should be exercised or else much valuable time and money will be wasted. Finally, it cannot be too strongly emphasised that cases which have a good chance of recovery should be treated along well-tried lines and not be subjected to excessive experimentation by an over-zealous doctor, lest they pass to the stage when treatment is of no avail. The fact remains that in our treatment of leprosy our resources must be almost limitless, for hardly any two cases react to exactly the same line of treatment, and it is impossible to outline all the possible methods. In the discussion to follow I hope this will be brought out.

\section{Results of treatment.}

It is extremely difficult to evaluate the results of antileprosy treatment, and the sceptic has ample opportunity of saying that no treatment has been definitely proved to be of any value. In assessing the results we must steer between the Scylla of excessive optimism and the Charybdis of extreme pessimism. I think a fair statement may be made that the modern therapeutics of leprosy will bring a large number of early cases to a state of complete freedom from the disease, and that the majority of infective cases, in the course of time, will become non-infective. In our endeavours to encourage workers we must not be blind to the fact that we are a very long way off a specific remedy for the disease, in the sense that arsenic is for syphilis, or quinine for malaria. Leprosy may become arrested not only in the late stage, the type which has long been known in India as " burnt-out," but also in the early stage.

If we admit that the so-called abortive case occurs in the early stage and that many late cases become selfarrested without treatment, there remain that large group of cases, chiefly in the cutaneous stage, which, without adequate treatment, would become more advanced, in this latter group are we able to assess the value of the treatment at all accurately. Percentages are difficult to estimate. In Purulia in 1932, the percentage of symptom-free cases 
among the in-patients was $6 \%$ and among the out-patients $3.8 \%$. Such results are not encouraging. Institutions such as Dichpali, where there are fewer advanced cutaneous cases, report better results, but how much is due to the better conditions rather than to any specific action of the drug used?

Again, the reports of the relapse rates both in Hawaii, and I believe now in Culion, are somewhat alarming, and therefore this question arises. In our treatment of cutaneous cases are we merely scarring up superficial lesions or are we getting at the deep foci of infection? We know as in tubercle, so in leprosy, when patients return to their former surroundings they are very liable to relapse, hence the importance of a settlement where they can gradually become accustomed to ordinary conditions of life.

One must confess that we have a long way to go in our search for better remedies for the disease. It may be that leprosy does not lend itself to specific treatment and that our greatest advance will be along epidomiological and preventive lines.

While expressing these doubts I do wish to emphasise most clearly that I am not pessimistic. I have seen a great change in the therapeutics of leprosy, but I am, as others are, only too much aware of the distance yet to be travelled, and therefore we must keep an open mind and be ever on the look out for new and better remedies. 